\title{
Relationship between adipocytokines and cardiovascular risk factors in patients with type 2 diabetes mellitus
}

\author{
SEMA USLU $^{1}$, NUR KEBAPÇI ${ }^{2}$, MEHMET KARA $^{1}$ and CENGIZ BAL ${ }^{3}$ \\ Departments of ${ }^{1}$ Medical Biochemistry, ${ }^{2}$ Endocrinology and ${ }^{3}$ Biostatistics, The Medical School, \\ Eskişehir Osmangazi University, Eskişehir 26480, Turkey
}

Received January 30, 2012; Accepted April 2, 2012

DOI: $10.3892 /$ etm.2012.557

\begin{abstract}
The aim of this study was to explore the relationship between serum profiles of adiponectin, leptin, resistin and visfatin and traditional and non-traditional cardiovascular risk factors in patients with type 2 diabetes mellitus (T2DM). A total of 85 patients with T2DM and 30 non-diabetic controls were enrolled in the study. Levels of adipocytokines (adiponectin, leptin, resistin and visfatin), lipids (total cholesterol, triglycerides), lipoproteins [HDL-cholesterol, LDL-cholesterol, lipoprotein (a)], apolipoproteins (Apo-A1 and Apo-B), non-traditional cardiovascular risk markers [asymmetric dimethylarginine (ADMA), homocysteine] and the inflammatory marker hs-CRP were measured, and anthropometric variables were determined. Serum adiponectin levels were decreased and leptin, resistin and visfatin levels were increased in T2DM patients compared to controls. They were associated with obesity (BMI), insulin resistance (HOMA-IR) and various markers of glucose/lipid profile, inflammation and endothelial dysfunction markers. These results suggest that decreased serum adiponectin and increased leptin, resistin and visfatin levels in T2DM may be novel biochemical risk factors for cardiovascular complications.
\end{abstract}

\section{Introduction}

Type 2 diabetes mellitus (T2DM) is a major risk factor for cardiovascular disease (CVD). The rising incidence of T2DM has resulted in CVD becoming the leading cause of morbidity and mortality worldwide (1). This increased risk of CVD is due to a complex cluster of risk factors associated with T2DM including insulin resistance, hyperglycemia, diabetic dyslipidemia, hypertension, hyperinsulinemia, systemic inflammation and adipose tissue-derived factors (2). Changes in the mass and metabolism of adipose tissue may be related to insulin resistance and visceral obesity commonly associated with T2DM (3).

Correspondence to: Dr Sema Uslu, Department of Medical Biochemistry, The Medical School, Eskişehir Osmangazi University, Eskişehir 26480, Turkey

E-mail: suslu@ogu.edu.tr

Key words: adipocytokines, cardiovascular risk markers, type 2 diabetes mellitus
Adipose tissue is no longer considered to be an inactive organ, which only stores lipids and serves as an energy reservoir. Numerous studies have shown that it is an active endocrine organ and secretes many substances that are involved in the regulation of several metabolic and physiologic processes. These chemical messengers, known as 'adipocytokines' or 'adipokines', include tumor necrosis factor $\alpha$ (TNF- $\alpha$ ), adiponectin, leptin, resistin and visfatin (4).

Adiponectin, also known as adipocyte complement-related protein (Acrp 30), gelatin-binding protein 28 (Gbp 28), adipose most abundant gene transcript (apM1) or adipo Q (5), is considered to be a protective protein with antidiabetic, antiinflammatory and anti-atherogenic effects (4). Reduced plasma adiponectin levels have been reported in obese individuals, particularly in those with visceral obesity, and have been negatively correlated with insulin resistance. Furthermore, decreased adiponectin levels were found to be associated with a higher incidence of T2DM (5). Leptin is the first identified endocrine product of adipose tissue and was found to regulate vascular function through local and central mechanisms (2). The primary role of leptin is to provide a signal to the central nervous system concerning the balance of body energy, which helps to control appetite and food intake, and to maintain stable body weight. However, leptin receptors are located throughout the body, suggesting that leptin is also involved in the regulation of various processes (4). There is also some evidence supporting the effects of leptin on the cardiovascular system. Leptin was shown to promote the development of atherosclerosis by inducing oxidative stress in endothelial cells, increasing platelet aggregation, and hypertrophy and proliferation of vascular smooth muscle cells (4). Additionally, it was shown that a high leptin level predicts subsequent development of T2DM (6). Resistin belongs to a family of cystein-rich secretory proteins called resistin-like molecules or FIZZ (found in inflammatory zones) proteins. The term 'resistin' comes from the development of insulin resistance in mice following injection of resistin. Resistin has been suggested to form a biochemical relationship between obesity and T2DM (7). Visfatin, which was originally termed pre- $\beta$ cell colony-enhancing factor (PBEF), enhances the maturation of $\beta$ cell precursors and displays nicotinamide phosphoribosyltransferase (Nampt) activity, and was found to be substantially correlated with the amount of visceral fat in humans (7). Although several clinical studies have analyzed the relationship of visfatin with insulin resistance, diabetes, 
Table I. Clinical characteristics of the type 2 diabetic patients and controls.

\begin{tabular}{|c|c|c|c|}
\hline & Controls & $\mathrm{T} 2 \mathrm{DM}$ & P-value \\
\hline Total no. & 30 & 85 & \\
\hline Gender (male/female) & $14 / 16$ & $40 / 45$ & \\
\hline Mean age (years) & $58.00 \pm 12.31$ & $61.76 \pm 11.67$ & NS \\
\hline Duration of DM (years) & - & $9.00(4.00-15.00)$ & - \\
\hline $\mathrm{BMI}\left(\mathrm{kg} / \mathrm{m}^{2}\right)$ & $27.37 \pm 4.21$ & $28.59 \pm 4.48$ & NS \\
\hline Glucose (mg/dl) & $81.50(76.00-86.25)$ & $140.00(116.50-187.50)$ & $<0.001$ \\
\hline HbA1c $(\%)$ & $4.15(3.8-5.20)$ & $7.76(6.69-6.79)$ & $<0.001$ \\
\hline Insulin $(\mu \mathrm{U} / \mathrm{ml})$ & $6.90(4.43-9.15)$ & $8.80(5.70-12.55)$ & $<0.001$ \\
\hline HOMA-IR & $1.37(0.90-1.97)$ & $3.05(1.90-5.06)$ & $<0.001$ \\
\hline $\mathrm{SBP}(\mathrm{mmHg})$ & $122.50(120.00-130.00)$ & $130.00(120.00-147.50)$ & $<0.001$ \\
\hline DBP (mmHg) & $80.00(73.50-81.25)$ & $80.00(72.50-90.00)$ & NS \\
\hline \multicolumn{4}{|l|}{ Medications } \\
\hline INS1/INS2/INS3 & - & $2 / 6 / 8$ & \\
\hline SU1/SU2/SU3/SU4/SU5 & - & $5 / 12 / 8 / 11 / 5$ & \\
\hline Metformin alone & - & 7 & \\
\hline Metformin and $\alpha$-GI & - & 11 & \\
\hline Diet alone & - & 10 & \\
\hline $\mathrm{A} / \mathrm{B} / \mathrm{C} / \mathrm{D} / \mathrm{E} / \mathrm{F} / \mathrm{G} / \mathrm{H} / \mathrm{I}$ & - & $6 / 5 / 4 / 8 / 6 / 12 / 7 / 5 / 14$ & \\
\hline Statins/Fiber & - & $25 / 14$ & \\
\hline ASA & - & 42 & \\
\hline
\end{tabular}

Values are expressed as the mean \pm SD or median (25th-75th percentiles). T2DM, type 2 diabetes mellitus; BMI, body mass index; HbA1C, glycosylated hemoglobin; SBP, systolic blood pressure; DBP, diastolic blood pressure; HOMA-IR, homeostatic model assessment index. INS (insulin): INS1 indicates INS alone; INS2, INS and $\alpha$-GI; INS3, INS, metformin and $\alpha$-GI. SU (sulfonilurea): SU1 indicates SU alone; SU2, SU and metformin; SU3, SU and $\alpha$-GI ( $\alpha$-glucosidase inhibitors); SU4, SU, metformin and $\alpha$-GI; SU5, SU and insulin. Antihypertensive drugs: $\mathrm{A}$, angiotensin converting enzyme inhibitor (ACE-i) alone; $\mathrm{B}$, angiotensin-II receptor blocker (ARB) alone; $\mathrm{C}$, calcium channel blocker (Ca) alone; D, Ca and ARB; E, Ca and, $\alpha$ - or $\beta$-blocker; F, Ca and ARB and/or ACE-i, $\alpha$ - or $\beta$-blocker; G, ACE-i and diuretics; H, ARB and diuretics; I, ACE-i and ARB and diuretics. ASA, acetylsalicylic acid. NS, not significant.

and obesity, its role in predicting diabetes remains unclear (8). Adipocytokines, as pro-inflammatory mediators, and several other inflammatory markers have been found to be elevated in obese subjects. Pro-inflammatory adipocytokines including adiponectin, leptin, resistin and visfatin seem to contribute to the 'low-grade inflammatory state' of obese individuals and to several metabolic disorders including cardiovascular complications (9). To our knowledge, there are few study concerning the relationship between adipocytokines and non-traditional cardiovascular risk factors such as homocysteine (Hcy) and asymmetric dimethyarginine (ADMA) levels.

Thus, the aim of this study was to explore the relationship of serum profiles of adipocytokines (adiponectin, leptin, resistin and visfatin) with traditional and non-traditional risk factors and with anthropometric variables in patients with T2DM.

\section{Materials and methods}

Study subjects. This study was performed at Eskişehir Osmangazi University, Faculty of Medicine in Turkey. Between January 2007 and March 2008, 85 subjects with T2DM were enrolled in the study. T2DM was diagnosed according to the American Diabetes Association Criteria. Of these patients, 45 had established cardiovascular disease and 40 had no evidence of CVD. Clinical evidence of CVD included myocardial infarction or coronary artery by-pass surgery $(n=17)$, stroke $(n=8)$, and peripheral arterial disease $(n=20)$. Patients in the group without vascular disease were T2DM patients who had no history of vascular disease, and those with normal ECG findings at exercise and normal peripheral artery Doppler ultrasonography findings. The clinical features of the patients are listed in Table I. All patients were receiving antidiabetic and antihypertensive therapies and some were receiving antilipidemic drugs and/or aspirin for at least the previous 6 months (Table I). Exclusion criteria were the presence of sustained type $1 \mathrm{DM}$, acute and chronic infections, malignancy, hepatic or renal disease, diabetic retinopathy and nephropathy, and other endocrine dysfunctions.

The control group consisted of 30 healthy control subjects with no history of T2DM, other endocrine dysfunctions, hyperlipidemia, hypertension, or coronary heart diseases. None of the subjects had received any medication (hormone replacement therapy, corticosteroids, vitamin supplements, antioxidant formulations and thiazolidinediones) which may have affected insulin resistance and/or endothelial function and none of the subjects were current smokers and consumers of alcohol. Blood pressure of all subjects was measured twice with a random zero mercury sphygmomanometer after 10 min of rest. 
Table II. Traditional and non-traditional cardiovascular risk markers of the study groups.

\begin{tabular}{lccc}
\hline & Controls & T2DM & P-value \\
\hline TC $(\mathrm{mg} / \mathrm{dl})$ & $171.50(162.00-185.25)$ & $192.00(168.00-231.50)$ & $<0.001$ \\
TG $(\mathrm{mg} / \mathrm{dl})$ & $119.00(98.50-144.25)$ & $200.00(138.50-268.50)$ & $<0.001$ \\
HDL-C $(\mathrm{mg} / \mathrm{dl})$ & $56.43 \pm 9.15$ & $41.45 \pm 9.64$ & $<0.001$ \\
LDL-C $(\mathrm{mg} / \mathrm{dl})$ & $92.00(80.45-105.70)$ & $112.80(85.00-145.80)$ & $<0.001$ \\
Apo-A1 $(\mathrm{mg} / \mathrm{dl})$ & $106.75(146.10-172.45)$ & $131.20(118.10-156.50)$ & $<0.001$ \\
Apo-B $(\mathrm{mg} / \mathrm{dl})$ & $95.45(78.45-103.03)$ & $109.80(91.40-122.90)$ & $<0.001$ \\
Lp $(\mathrm{a})(\mathrm{mg} / \mathrm{dl})$ & $21.25 \pm 5.14$ & $45.07 \pm 16.37$ & $<0.001$ \\
$\mathrm{hs}-\mathrm{CRP}(\mathrm{mg} / \mathrm{dl})$ & $0.30(0.18-0.40)$ & $1.45(0.87-2.85)$ & $<0.001$ \\
Hcy $(\mathrm{mg} / \mathrm{dl})$ & $7.67 \pm 1.86$ & $10.92 \pm 3.39$ & $<0.001$ \\
ADMA $(\mu \mathrm{g} / \mathrm{ml})$ & $0.56(0.54-0.70)$ & $0.76(0.59-0.99)$ & $<0.05$ \\
L/A ratio & $0.58(0.47-0.86)$ & $2.01(1.03-3.82)$ & $<0.001$
\end{tabular}

Values are expressed as mean \pm SD or median (25th-75th percentiles). T2DM, type 2 diabetes mellitus; TC, total cholesterol; TG, triglycerides; HDL-C, high-density lipoprotein-cholesterol; LDL-C, low-density lipoprotein-cholesterol; Apo-A1, apolipoprotein A1; Apo-B, apolipoprotein B; Lp (a), lipoprotein (a); Hcy, homocysteine; ADMA, asymetric dimethylarginine; L/A ratio, leptin/adiponectin ratio.

All subjects were informed in regards to the aim of the study. The study was approved by the Ethics Committee of Eskişehir Osmangazi University Medical Faculty (2006/638).

Blood sample collection. Overnight fasting blood samples were drawn from the patients and controls between 7.00 and 9.00 a.m. via the venipuncture of an antecubital vein. Blood samples were collected in vacutainer tubes with a gel separator and in heparinized tubes for $\mathrm{HbA} 1 \mathrm{C}$ measurements and were centrifugated at $2000 \mathrm{rpm}$ for $15 \mathrm{~min}$ at $4^{\circ} \mathrm{C}$ after an incubation period of $30 \mathrm{~min}$. All biochemical variables were measured on the same day of the blood collection. Remaining serum specimens were stored at $-20^{\circ} \mathrm{C}$ until analysis of adiponectin, leptin, resistin, visfatin and ADMA levels.

Biochemical analyses. Serum glucose, total cholesterol (TC), HDL-cholesterol (HDL-C), triglyceride (TG) were measured by an enzymatic colorimetric method and Apo-A1, Apo-B, lipoprotein (a) and hs-CRP levels using an immunoassay method on a Hitachi Modular analyzer using Roche Diagnostic kits. Insulin levels were measured by chemiluminescence immunoassay method using DPC Immulite-I analyzer (Diagnostic Products Corp., Los Angeles, CA, USA) kits. HbA1C levels were measured by a turbidimetry immunoassay method using Hitachi Modular analyzer with Roche Diagnostic kits. Serum homocystein levels were measured using DPC kits on an Immulite-2000 autoanalyzer.

Serum adiponectin levels were measured by enzyme linked immunosorbent assay (ELISA) using AviBion Human Adiponectin (Orgenium Laboratories, Helsinki), with a sensitivity of $3 \mathrm{ng} / \mathrm{ml}$. Serum leptin levels were measured by an active human leptin ELISA (DSL, Diagnostic System Laboratories, USA) with a sensitivity of $0.05 \mathrm{ng} / \mathrm{ml}$. Serum resistin levels were measured by ELISA (Linco Research, USA) with a sensitivity of $0.16 \mathrm{ng} / \mathrm{ml}$. Serum visfatin levels were measured by ELISA using the Biosource Immunoassay test (Biosource International, Inc., USA) with a sensitivity of $602 \mathrm{pg} / \mathrm{ml}$. Asymmetric dimethylarginine (ADMA) was measured using an ELISA kit (Immundiagnostik AG, Bensheim) with a sensitivity $0.05 \mu \mathrm{g} / \mathrm{ml}$.

Calculations. Low-density lipoprotein-cholesterol (LDL-C) was calculated using the Friedewald formula. Standing height and body weight were measured with the subjects dressed in light indoor clothing without shoes. Body mass index (BMI) was calculated as weight divided by the square of the height $\left(\mathrm{kg} / \mathrm{m}^{2}\right)$. In order to determine insulin resistance, HOMA-IR index (Homeostasis model assessment) was calculated as: HOMA $=[($ fasting serum glucose $(\mathrm{mmol} / \mathrm{l}) \mathrm{x}$ fasting insulin $(\mu \mathrm{U} / \mathrm{ml}) / 22.5]$. Another criteria for insulin resistance, the ratio of leptin/adiponectin (L/A), was also calculated.

Statistical analysis. Statistical analysis was performed using SPSS for Windows (Statistical Package for the Social Sciences, version 20.0; SSPS Inc. Chicago, IL, USA). The normal distribution of the variables was evaluated using the Shapiro-Wilk test. The Mann-Whitney U test was used for the comparison of variables which were not normally distributed, and the independent Student's t-test for the comparison of variables which were normally distributed. The Pearson and Spearman tests were used for the evaluation of correlations among the variables according to the distribution of variables. $\mathrm{P}<0.05$ was accepted as indicative of statistical significance. The results of variables with a normal distribution were expressed as mean \pm SD and those with a non-Gaussian distribution were expressed as median (25th-75th percentile).

\section{Results}

Subject characteristics and anthropometric variables are summarized in Table I. There were no significant differences in age, BMI and DBP levels between the T2DM patients and controls $(\mathrm{P}>0.05)$. In the T2DM patients, glucose, insulin, HbA1C, HOMA-IR and SBP levels were significantly higher than the values in the controls $(\mathrm{P}<0.001$, for all). Table II summarizes blood lipid parameters and non-traditional CVD 


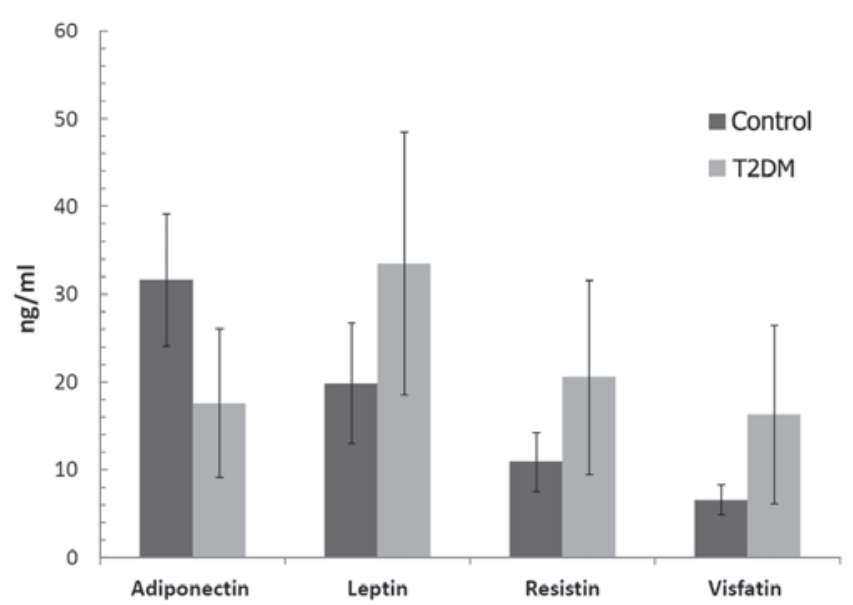

Figure 1. Comparison of adiponectin, leptin, resistin and visfatin levels in the T2DM patients and control subjects.

markers. T2DM patients had significantly higher TC, LDL-C, TG, Lp (a), Apo-A1, Apo-B, Hcy and ADMA levels, and L/A ratios than the controls $(\mathrm{P}<0.001$ for each comparison; for ADMA $\mathrm{P}<0.05$ ).

Fig. 1 illustrates the comparison of adiponectin, leptin, resistin and visfatin levels in the T2DM patients and control subjects. Serum adiponectin levels were decreased significantly $(\mathrm{P}<0.001)$, while leptin, resistin and visfatin levels were increased in the T2DM patients compared to these levels in the controls ( $\mathrm{P}<0.001$, for each comparison).

Table III represents the relationship between serum adiponectin, leptin, resistin and visfatin levels and anthropometric variables. Adiponectin was significantly and negatively correlated with HbA1C levels, BMI, HOMA-IR, SBP and DBP. Leptin was positively correlated with age, BMI, glucose and insulin levels, HOMA-IR, SBP and DBP. Resistin was positively correlated with DM duration, BMI, HOMA-IR, SBP and DBP. Visfatin was positively correlated with BMI, insulin, HbA1C, HOMA-IR, SBP and DBP levels.

Table IV represents the relationship between serum levels of adiponectin, leptin, resistin and visfatin and traditional and non-traditional CVD risk markers in T2DM patients. Adiponectin was positively correlated with TG, LDL-C, Apo-A1, Hcy, ADMA and TG, hs-CRP, DBP and L/A ratio and negatively with HDL-C. Leptin was positively correlated with TG, Lp (a), Apo-A1, Apo-B, hs-CRP, Hcy, ADMA and L/A ratio and negatively correlated with HDL-C. Resistin was positively with TC, TG, Apo-A1, Apo-B, hs-CRP, Hcy and L/A ratio and negatively correlated with HDL-C. Visfatin was correlated positively with TC, TG, LDL-C, hs-CRP, Hcy and L/A ratio.

\section{Discussion}

During the past few years, much attention has been focused on the potential role of adipose tissue in the development of vascular complications of diabetes. This study was designed to explore the relationship between adipocytokines and traditional and non-traditional risk markers and with anthropometric measurements in T2DM. Adiponectin, an adipocytokine secreted by fat cells, has regulatory functions on energy metabolism; its low levels are predictive of future development of T2DM. Moreover, it is likely to have a central role in the pathogenesis of T2DM (10).

In a recent meta-analysis, $\mathrm{Li}$ et al observed a significant inverse relationship between plasma adiponectin levels and the incidence of T2DM. Risk of T2DM appeared to decrease with increasing adiponectin levels. Currently, adiponectin is one of the strongest biochemical predictors of T2DM (11). In this study, diabetic patients showed significantly decreased serum adiponectin levels compared to the controls. Also, serum levels of lipids and lipoproteins (TG, LDL-C, Apo-A1 and Apo-B) were significantly increased but HDL-C was decreased with decreasing adiponectin levels, as reported in previous studies (12-14). Several studies have also reported a positive correlation between plasma adiponectin and HDL-C levels (15). These data strongly suggest a possible direct link between adiponectin and HDL-C. Moreover, Apo-A1 and HDL-C levels were found to be inversely correlated with the incidence of metabolic syndrome, which is associated with an excess risk of T2DM and cardiovascular disease (16). Low adiponectin levels and increased HDL core TG content resulting from increased neutral lipid exchange between triglyceride-rich lipoproteins and HDL in hypertriglyceridemic, insulin-resistant states may decrease HDL particle numbers and cholesterol content, potentially by enhancing catabolism of HDL particles $(15,17)$. Furthermore, we found a correlation between serum levels of adiponectin and increased LDL-C levels in T2DM patients. Lautamäki et al suggest that antioxidant effects of adiponectin on LDL particles

Table III. Relationship between adiponectin, leptin, resistin and visfatin and anthropometric variables ${ }^{\mathrm{a}}$.

\begin{tabular}{lccccccrrr}
\hline & $\begin{array}{c}\text { Age } \\
(\text { years })\end{array}$ & $\begin{array}{c}\text { DM duration } \\
\text { (years })\end{array}$ & $\begin{array}{c}\text { BMI } \\
\left(\mathrm{kg} / \mathrm{m}^{2}\right)\end{array}$ & HOMA-IR & $\begin{array}{c}\text { Glucose } \\
(\mathrm{mg} / \mathrm{dl})\end{array}$ & $\begin{array}{c}\text { Insulin } \\
(\mu \mathrm{U} / \mathrm{l})\end{array}$ & $\begin{array}{c}\text { HbA1C } \\
(\%)\end{array}$ & $\begin{array}{c}\text { SBP } \\
(\mathrm{mmHg})\end{array}$ & $\begin{array}{c}\text { DBP } \\
(\mathrm{mmHg})\end{array}$ \\
\hline $\mathrm{A},(\mathrm{ng} / \mathrm{ml})$ & -0.18 & -0.13 & $-0.32^{\mathrm{c}}$ & $-0.26^{\mathrm{b}}$ & -0.13 & -0.20 & $-0.22^{\mathrm{b}}$ & $-0.27^{\mathrm{b}}$ & $-0.26^{\mathrm{b}}$ \\
$\mathrm{L},(\mathrm{ng} / \mathrm{ml})$ & $0.30^{\mathrm{b}}$ & 0.21 & $0.37^{\mathrm{c}}$ & $0.37^{\mathrm{c}}$ & $0.24^{\mathrm{b}}$ & $0.34^{\mathrm{c}}$ & $0.24^{\mathrm{b}}$ & $0.22^{\mathrm{b}}$ & $0.24^{\mathrm{b}}$ \\
$\mathrm{R},(\mathrm{ng} / \mathrm{ml})$ & -0.21 & -0.12 & $-0.30^{\mathrm{c}}$ & $-0.25^{\mathrm{b}}$ & -0.14 & -0.20 & -0.14 & $-0.31^{\mathrm{c}}$ & $-0.26^{\mathrm{b}}$ \\
$\mathrm{V},(\mathrm{ng} / \mathrm{ml})$ & -0.19 & -0.14 & $-0.23^{\mathrm{b}}$ & $-0.26^{\mathrm{b}}$ & -0.17 & $-0.26^{\mathrm{b}}$ & $-0.27^{\mathrm{b}}$ & $-0.21^{\mathrm{b}}$ & $-0.26^{\mathrm{b}}$ \\
\hline
\end{tabular}

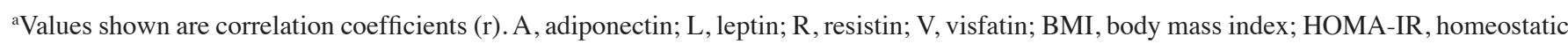
model assessment index; HbA1C, glycosylated hemoglobin; SBP, systolic blood pressure; DBP, diastolic blood pressure. ${ }^{b} \mathrm{P}<0.05$, ${ }^{\mathrm{C}}<0.01$. 
Table IV. Relationship between adiponectin, leptin, resistin and visfatin with traditional and non-traditional cardiovascular risk markers $^{\mathrm{a}}$.

\begin{tabular}{|c|c|c|c|c|}
\hline & Adiponectin (ng/ml) & Leptin (ng/ml) & Resistin (ng/ml) & Visfatin (ng/ml) \\
\hline $\mathrm{TC}(\mathrm{mg} / \mathrm{dl})$ & -0.17 & 0.13 & $0.24^{\mathrm{b}}$ & $0.37^{\mathrm{c}}$ \\
\hline $\mathrm{TG}(\mathrm{mg} / \mathrm{dl})$ & $-0.35^{\mathrm{c}}$ & $0.33^{\mathrm{c}}$ & $0.25^{\mathrm{b}}$ & $0.36^{\mathrm{c}}$ \\
\hline HDL-C (mg/dl) & $0.36^{\mathrm{c}}$ & $-0.25^{\mathrm{b}}$ & $-0.28^{\mathrm{b}}$ & -0.16 \\
\hline LDL-C (mg/dl) & $-0.10^{c}$ & 0.14 & 0.13 & $0.30^{\mathrm{c}}$ \\
\hline $\mathrm{Lp}$ (a) (mg/dl) & -0.17 & $0.23^{\mathrm{b}}$ & 0.11 & 0.19 \\
\hline Apo-A1 (mg/dl) & $-0.30^{c}$ & $0.24^{\mathrm{b}}$ & $0.21^{\mathrm{b}}$ & 0.20 \\
\hline Apo-B (mg/dl) & -0.12 & 0.16 & $0.24^{\mathrm{b}}$ & 0.11 \\
\hline hs-CRP (mg/dl) & $-0.30^{\mathrm{c}}$ & $0.27^{\mathrm{b}}$ & $0.26^{\mathrm{b}}$ & $0.31^{\mathrm{c}}$ \\
\hline Hcy (mg/dl) & $-0.26^{\mathrm{b}}$ & $0.30^{c}$ & $0.23^{\mathrm{b}}$ & $0.25^{\mathrm{b}}$ \\
\hline ADMA $(\mu \mathrm{g} / \mathrm{ml})$ & $0.25^{\mathrm{b}}$ & $0.24^{\mathrm{b}}$ & 0.10 & 0.16 \\
\hline $\mathrm{L} / \mathrm{A}$ ratio & $-0.77^{\mathrm{c}}$ & $0.63^{c}$ & $0.45^{\mathrm{c}}$ & $0.49^{c}$ \\
\hline Visfatin (ng/ml) & $-0.55^{\mathrm{c}}$ & $0.52^{\mathrm{c}}$ & $0.39^{c}$ & - \\
\hline Resistin (ng/ml) & $-0.57^{c}$ & $0.43^{c}$ & - & \\
\hline Leptin $(\mathrm{ng} / \mathrm{ml})$ & $-0.55^{\mathrm{c}}$ & - & & \\
\hline
\end{tabular}

${ }^{a}$ Values shown are correlation coefficients (r). TC, total cholesterol; TG, triglycerides; HDL-C, high-density lipoprotein-cholesterol; LDL-C, low-density lipoprotein-cholesterol; Apo-A1, apolipoprotein A1; Apo-B, apolipoprotein B; Lp (a), lipoprotein (a); Hcy, homocysteine; ADMA, asymetric dimethylarginine; $\mathrm{L} / \mathrm{A}$ ratio, leptin/adiponectin ratio. ${ }^{\mathrm{b}} \mathrm{P}<0.05,{ }^{\mathrm{c}} \mathrm{P}<0.01$.

in the arterial wall may result in decreased production of oxidized LDL lipoprotein (OxLDL), which may be beneficial in patients with coronary artery disease by promoting plaque stability (12).

Serum adiponectin levels were found to be negatively correlated with BMI and HbA1C levels and no significant correlation was found between serum adiponectin levels and age, DM duration, glucose and insulin levels, as reported in previous studies (12). In the present study, we also examined the association between adipocytokines and hs-CRP, an inflammatory marker. Chronic inflammation is likely to play a role in the pathogenesis of T2DM. We found that adiponectin was negatively correlated with hs-CRP levels. Krakoff et al have revealed that adiponectin may be an important link between adiposity and inflammation and T2DM (18). Elevated serum hs-CRP may play a role in the development of insulin resistance syndrome and T2DM. However, this elevation is accompanied by decreased levels of adiponectin, suggesting an anti-inflammatory effect of adiponectin. Since adiponectin could counteract the pro-inflammatory effects of TNF- $\alpha$ in vascular cell components and adipose tissue, it may also influence IL- 6 and CRP production through the modulation of TNF- $\alpha$ action. Therefore, adiponectin may directly or indirectly affect CRP levels in plasma and adipose tissue through modulating inflammatory cascades (19).

There is a growing body of evidence that leptin is an independent risk factor for CVD and is likely to be an important link in the development of cardiovascular risk and obesity (20). Leptin plays an important role in the long-term regulation of body weight. Paradoxically, markedly increased plasma leptin levels were found in obese individuals, suggesting a resistance to its effects on target organs when produced excessively (21). We found that increased leptin levels were correlated with $\mathrm{BMI}$ and insulin resistance (HOMA-IR) in T2DM patients, as reported in previous studies $(20,21)$. Insulin resistance was reported to contribute to hyperleptinemia indirectly (22). Abdella et al reported that the hyperinsulinemia that frequently accompanies obesity is likely to result in increased ob gene expression and higher plasma leptin levels. Therefore, the association found between leptin and insulin may simply reflect the size of adipose tissue stores (23). High leptin levels generally associated with high insulin levels could be partially explained by resistance to leptin such that chronically elevated leptin levels in obesity may result in decreased responsiveness of the receptor system in pancreatic $\beta$ cells, leading to increased insulin secretion. The resulting hyperinsulinemia in turn could exacerbate obesity and further increase leptin levels, resulting in a positive feedback loop that could promote the development of diabetes (24,25). Accordingly, we found a close relationship between insulin and leptin levels in T2DM patients. Additionally, leptin levels positively correlated with TG, Lp (a), Apo-A1, glucose, SBP and DBP levels and negatively with HDL-C levels in T2DM patients. Notably, hyperleptinemia in obesity is suggested to dysregulate blood pressure that results in hypertension, suggesting that leptin may be a potential predictor of hypertension (26).

Another potential physiological role for leptin is that the leptin-mediated T-cell immune response stimulates proliferation of T-helper cells and increases production of pro-inflammatory cytokines through regulation of immune cells (20). We found an association between serum leptin levels and hs-CRP, an inflammatory marker that is related to both metabolic syndrome and cardiovascular events. Adipose tissue is the source of circulating leptin and also synthesizes IL-6, which induces CRP synthesis in the liver. Leptin may also directly induce production of IL-6, resulting in further upregulation of hepatic CRP production (20). Thus, serum leptin levels may be used as an integrated marker of adiposity, 
insulin resistance and vascular dysfunction that will be useful in clinical practice for cardiovascular risk stratification. In addition, leptin and adiponectin have opposing effects on subclinical inflammation. Leptin upregulates cytokines such as tumor necrosis factor- $\alpha(\mathrm{TNF}-\alpha)$ and IL-6, that are associated with insulin resistance in T2DM and it can be considered as a pro-inflammatory cytokine. In contrast, adiponectin downregulates the expression and release of many pro-inflammatory immune mediators and exerts anti-inflammatory properties (21). Thorand et al indicated that the two adipokines, leptin and adiponectin, interact with each other in the modulation of T2DM risk, but adiponectin is likely to have a stronger association with T2DM risk compared to leptin (25). We also found an inverse relationship between leptin and adiponectin in T2DM patients. Although leptin or adiponectin was separately associated with the risk of T2DM, the association of T2DM risk with leptin/adiponectin ratio (L/A) was stronger than that with leptin alone or with adiponectin alone (27). Accordingly, Oda et al suggested that the L/A ratio may be a useful index for insulin resistance in clinical practice. In addition, it may also be a good indicator for assessing the effectiveness of antidiabetic therapy. We, therefore, calculated the HOMA-IR index and L/A ratio to reveal insulin resistance. As expected, adiponectin and leptin were correlated with L/A ratio negatively and positively, respectively.

Resistin is another protein secreted by adipocytes and leads to insulin resistance in vitro and in vivo, constituting a possible link between obesity and diabetes (28). In the present study, we found markedly higher serum resistin levels in T2DM patients compared to controls. Mean serum resistin levels were correlated positively with BMI and insulin resistance (as assessed by HOMA-IR and the L/A ratio). The initial concept of resistin being the link between obesity, insulin resistance and diabetes is currently debated (29). Mojiminiyi et al found that resistin is positively associated with markers of obesity, inflammation and insulin resistance but negatively correlated with markers of insulin sensitivity. Furthermore, these associations were likely to be dependent on BMI, suggesting a possible link between resistin levels and the insulin resistance and low-grade inflammation accompanying obesity (30). As resistin may have a role in the development of insulin resistance, serum resistin levels could be a surrogate marker for the determination of insulin resistance (31).

In the present study, serum resistin levels were positively correlated with BMI, HOMA-IR, SBP, DBP, TC, TG, Lp (a), Apo-A1, leptin and negatively correlated with HDL-C and adiponectin levels in patients with T2DM. There were no correlations with age, DM duration, glucose, insulin and HbA1c levels $(28,29)$. Hyperresistinemia would contribute to the pathogenesis of hypertension in patients with T2DM (31). On the other hand, resistin levels were related with hs-CRP, similar to the results of Mojiminiyi et al suggesting a possible link between resistin and obesity and insulin resistance via pro-inflammatory pathways (29). Collectively, these data suggest that resistin is related to the cardiovascular inflammatory state in humans. Accordingly, CRP production induced by resistin may explain the correlation between obesity (BMI) and resistin levels (33). Several studies have shown that proinflammatory cytokines such as IL- $6, \mathrm{TNF}-\alpha$, leptin and possibly resistin induce CRP production (34). The positive correlation found between leptin and resistin in our study may also be explained by the same mechanism. We also found a negative correlation between serum adiponectin and resistin levels. Lau et al reported similar results and also suggested that the novel adiponectin-resistin (AR) and insulin resistance (IRAR) indices are cost-effective, precise, reproducible and reliable measures of insulin sensitivity (35). In addition, resistin has a pro-inflammatory role, which was shown to stimulate several factors, such as ET-1 (endothelin-1), VCAM-1 (vascular cell adhesion molecule-1), and MCP-1 (monocyte chemoattractant protein-1). Several studies have suggested resistin as a cardiovascular risk factor and examined its role in endothelial dysregulation and atherosclerotic lesion formation (9).

Visfatin is a newly discovered adipocytokine secreted by intra-abdominal adipose tissue. Several clinical studies have analyzed the relationship between visfatin and insulin resistance, diabetes and obesity. However, these studies have provided disparate results $(9,36,37)$. In a meta-analysis, Chang et al suggested that the use of visfatin may predict obesity, diabetes status, insulin resistance, metabolic syndrome and cardiovascular disease (38). In this study, we found increased visfatin levels in T2DM patients, as reported in previous studies $(39,40)$. In addition, visfatin levels were positively correlated with HOMA-IR, L/A ratio and insulin levels in T2DM patients (41). El-Masallamy et al provided several explanations for the increased visfatin levels noted in patients with T2DM. Firstly, increased visfatin levels in diabetic patients may be due to impaired visfatin signaling in target tissues. Secondly, due to the insulin mimetic effects, increased plasma visfatin levels could be a compensatory mechanism in response to hyperglycemia that ameliorates the functional consequences of insulin deficiency or resistance. Thirdly, visfatin-mediated NAD biosynthesis that regulates glucose-stimulated insulin secretion may explain increased levels of visfatin in T2DM patients as a compensatory mechanism for $\beta$-cell functioning. Finally, because of the pro-inflammatory properties, these elevated levels could be attributed to the chronic low-grade inflammation present in T2DM (39).

On the other hand, a direct or indirect relationship may exist between visfatin and lipid metabolism. The relationship of visfatin with lipid profile might be a compensatory mechanism for diabetic dyslipidemia, since visfatin upregulates peroxisome proliferator-activated receptor $\gamma$ activity (39). We found that visfatin levels were positively correlated with markers of lipid metabolism such as TC, TG and LDL-C, as reported in previous studies (42). Also, serum glucose was an independent predictor of serum visfatin levels. Consistent with our findings, Sandeep et al showed that the visfatin secretion from adipocytes depends on the duration and extent of hyperglycemia (40). Accordingly, Shaker et al reported that high visfatin levels were positively related to glycemic control (41). Therefore, higher glucose and HbA1C levels may contribute to, at least partly, the increased visfatin levels found in T2DM patients.

Several studies have demonstrated that peripheral insulin resistance plays an important role in the development of endothelial dysfunction and cardiovascular disease. In addition, impaired nitric oxide (NO) bioavailability may also be responsible for the vascular complications noted in T2DM (43). At this point, asymmetric dimethylarginine (ADMA), the major endogenous inhibitor of NO synthase (NOS), may be suggested as a 
causative factor for endothelial dysfunction. Increased plasma levels of ADMA are associated with several clinical conditions involving endothelial dysfunction, including hypertension, hypercholesterolemia, diabetes mellitus and cardiovascular disease $(44,45)$. Hyperglycemia and poor glycemic control may be the underlying mechanism of endothelial dysfunction in T2DM (46). Hyperglycemia impairs dimethylarginine dimethylaminohydrolase (DDAH) and causes the accumulation of ADMA, resulting in disturbances in NO pathways in vessels (46). We found increased ADMA levels in T2DM patients and the correlation of glucose, insulin and HbA1C levels with ADMA levels (data not shown). Notably, ADMA levels were correlated with hypoadiponectinemia and hyperleptinemia but not with hyperresistinemia and hypervisfatinemia. In fact, adiponectin and leptin increases NO production in vascular endothelium and inhibits endothelial cell activation, and both effects would be impaired due to decreased adiponectin levels, leptin resistance and/or increased ADMA levels. Because of the correlation found between all adipocytokines and systolic and diastolic blood pressures, we also expected to find a correlation between ADMA levels and visfatin and resistin, but there was no such correlation.

Another factor that can affect endothelial function is hyperhomocysteinemia (HHcy) (43). High Hcy levels have been associated with an increased risk of stroke and other CV events (47). In the present study, T2DM patients had higher Hcy levels than the controls, similar to the results reported by Akalin et al (48). Emerging evidence suggests that hyperhomocysteinemia is associated with adipose tissue dysfunction. Wang et al showed that Hcy inhibits lipolysis in adipocytes through AMPK activation and also reported that exogenous Hcy in the diet for 2 weeks lowered circulating glycerol and FFA levels. Moreover, Hcy supplementation was associated with increased leptin levels and decreased adiponectin levels in plasma (49). We found that Hcy levels were correlated positively with leptin, resistin and visfatin levels and negatively correlated with adiponectin level. HHcy may promote insulin resistance through increased resistin secretion from adipocytes via the activation of reactive oxygen species (ROS)-protein kinase $\mathrm{C}(\mathrm{PKC})$-nuclear factor (NF)- $\kappa \beta$ pathway (50).

The results of the present study demonstrated that secretion of adiponectin leptin, resistin and visfatin is altered in subjects with T2DM clearly suggesting that they may be related to obesity, hypertension and cardiovascular disease. These adipocytokines may be an important link between increased fat mass, insulin resistance, disorders of lipid and glucose metabolism and endothelial dysfunction in diabetic patients. Future studies on these adipocytokines may shed new light on the prevention and treatment of T2DM, and offer a new field for the development of novel drugs with which to alleviate insulin resistance and obesity. In summary, these results suggest that decreased serum adiponectin and increased leptin, resistin and visfatin levels in T2DM may be novel biochemical risk factors for cardiovascular complications.

\section{Acknowledgements}

This study was supported by grants from the Scientific Research Projects Commission of Eskişehir Osmangazi University (2006-11031).

\section{References}

1. Bartels DW, Davidson MH and Gong WC: Type 2 diabetes and cardiovascular disease: reducing the risk. J Manag Care Pharm 13: S2-S15, 2007.

2. Bakker W, Eringa EC, Sipkema P and van Hinsbergh VW: Endothelial dysfunction and diabetes: roles of hyperglycemia, impaired insulin signaling and obesity. Cell Tissue Res 335: 165-189, 2009.

3. Lebovitz HE: Insulin resistance - a common link between type 2 diabetes and cardiovascular disease. Diabetes Obes Metab 8: 237-249, 2006.

4. Kowalska I: Role of adipose tissue in the development of vascular complications in type 2 diabetes mellitus. Diabetes Res Clin Pract 78: 14-22, 2007.

5. Kadowaki T, Yamauchi T, Kubota N, Hara K, Ueki K and Tobe K: Adiponectin and adiponectin receptors in insulin resistance, diabetes, and the metabolic syndrome. J Clin Invest 116: 1784-1792, 2006

6. Yanagawa T, Taniguchi A, Fukushima M, Nakai Y, Nagasaka S, Ohgushi M, Matsumoto K, Kuroe A, Ohya M and Seino Y: Leptin, triglycerides, and interleukin 6 are independently associated with $\mathrm{C}$-reactive protein in Japanese type 2 diabetic patients. Diabetes Res Clin Pract 75: 2-6, 2007.

7. Rabe K, Lehrke M, Parhofer KG and Broedl UC. Adipokines and insulin resistance. Mol Med 14: 741-751, 2008.

8. Gulcelik NE, Usman A and Gürlek A: Role of adipocytokines in predicting the development of diabetes and its late complications. Endocrine 36: 397-403, 2009.

9. Gualillo O, González-Juanatey JR and Lago F: The emerging role of adipokines as mediators of cardiovascular function: physiologic and clinical perspectives. Trends Cardiovasc Med 17: 275-283, 2007.

10. Snehalatha C, Mukesh B, Simon M, Viswanathan V, Haffner SM and Ramachandran A: Plasma adiponectin is an independent predictor of type 2 diabetes in Asian indians. Diabetes Care 26: 3226-3229, 2003.

11. Li S, Shin HJ, Ding EL and van Dam RM: Adiponectin levels and risk of type 2 diabetes: a systematic review and meta-analysis. JAMA 302: 179-188, 2009.

12. Lautamäki R, Rönnemaa T, Huupponen R, Lehtimäki T, Iozzo $P$, Airaksinen KE, Knuuti J and Nuutila P: Low serum adiponectin is associated with high circulating oxidized low-density lipoprotein in patients with type 2 diabetes mellitus and coronary artery disease. Metabolism 56: 881-886, 2007.

13. Jaleel F, Jaleel A, Aftab J and Rahman MA: Relationship between adiponectin, glycemic control and blood lipids in diabetic type 2 postmenopausal women with and without complication of ischemic heart disease. Clin Chim Acta 370: 76-81, 2006.

14. Hotta K, Funahashi T, Arita Y, Takahashi M, Matsuda M, Okamoto Y, Iwahashi H, Kuriyama H, Ouchi N, Maeda K, et al: Plasma concentrations of a novel, adipose-specific protein, adiponectin, in type 2 diabetic patients. Arterioscler Thromb Vasc Biol 20: 1595-1599, 2000.

15. Vergès B, Petit JM, Duvillard L, Dautin G, Florentin E, Galland F and Gambert P: Adiponectin is an important determinant of apoA-I catabolism. Arterioscler Thromb Vasc Biol 26: 1364-1369, 2006.

16. Han R, Lai R, Ding Q, Wang Z, Luo X, Zhang Y, Cui G, He J, Liu W and Chen Y: Apolipoprotein A-I stimulates AMP-activated protein kinase and improves glucose metabolism. Diabetologia 50: 1960-1968, 2007.

17. Schulze MB, Shai I, Rimm EB, Li T, Rifai N and Hu FB: Adiponectin and future coronary heart disease events among men with type 2 diabetes. Diabetes 54: 534-539, 2005.

18. Krakoff J, Funahashi T, Stehouwer CD, Schalkwijk CG, Tanaka S, Matsuzawa Y, Kobes S, Tataranni PA, Hanson RL, Knowler WC and Lindsay RS: Inflammatory markers, adiponectin, and risk of type 2 diabetes in the Pima Indian. Diabetes Care 26: 1745-51, 2003.

19. Yuan G, Zhou L, Tang J, Yang Y, Gu W, Li F, Hong J, Gu Y, Li X, Ning G and Chen M: Serum CRP levels are equally elevated in newly diagnosed type 2 diabetes and impaired glucose tolerance and related to adiponectin levels and insulin sensitivity. Diabetes Res Clin Pract 72: 244-250, 2006.

20. Wannamethee SG, Tchernova J, Whincup P, Lowe GD, Kelly A, Rumley A, Wallace AM and Sattar N: Plasma leptin: associations with metabolic, inflammatory and haemostatic risk factors for cardiovascular disease. Atherosclerosis 191: 418-426, 2007. 
21. Reilly MP, Iqbal N, Schutta M, Wolfe ML, Scally M, Localio AR, Rader DJ and Kimmel SE: Plasma leptin levels are associated with coronary atherosclerosis in type 2 diabetes. J Clin Endocrinol Metab 89: 3872-3878, 2004.

22. Stefanović A, Kotur-Stevuljević J, Spasić S, Bogavac-Stanojević N and Bujisić $\mathrm{N}$ : The influence of obesity on the oxidative stress status and the concentration of leptin in type 2 diabetes mellitus patients. Diabetes Res Clin Pract 79: 156-163, 2008.

23. Abdella NA, Mojiminiyi OA, Moussa MA, Zaki M, Al Mohammedi H, Al Ozairi ES and Al Jebely S: Plasma leptin concentration in patients with type 2 diabetes: relationship to cardiovascular disease risk factors and insulin resistance. Diabet Med 22: 278-285, 2005

24. Asakawa H, Tokunaga K and Kawakami F: Relationship of leptin level with metabolic disorders and hypertension in Japanese type 2 diabetes mellitus patients. J Diabetes Complications 15: 57-62, 2001.

25. Thorand B, Zierer A, Baumert J, Meisinger C, Herder C and Koenig W: Associations between leptin and the leptin/adiponectin ratio and incident type 2 diabetes in middle-aged men and women: results from the MONICA/KORA Augsburg study 1984-2002. Diabet Med 27: 1004-1011, 2010.

26. Maenhaut N and van de Voorde J: Regulation of vascular tone by adipocytes. BMC Med 16: 9-25, 2011.

27. Oda N, Imamura S, Fujita T, Uchida Y, Inagaki K, Kakizawa H, Hayakawa N, Suzuki A, Takeda J, Horikawa Y and Itoh M: The ratio of leptin to adiponectin can be used as an index of insulin resistance. Metabolism 57: 268-273, 2008.

28. Lu HL, Wang HW, Wen Y, Zhang MX and Lin HH: Roles of adipocyte derived hormone adiponectin and resistin in insulin resistance of type 2 diabetes. World J Gastroenterol 12: 1747-1751, 2006.

29. Mojiminiyi OA and Abdella NA: Associations of resistin with inflammation and insulin resistance in patients with type 2 diabetes mellitus. Scand J Clin Lab Invest 67: 215-225, 2007.

30. Kusminski CM, McTernan PG and Kumar S: Role of resistin in obesity, insulin resistance and Type II diabetes. Clin Sci (Lond) 109: 243-256, 2005.

31. Tokuyama Y, Osawa H, Ishizuka T, Onuma H, Matsui K, Egashira T, Makino $\mathrm{H}$ and Kanatsuka A: Serum resistin level is associated with insulin sensitivity in Japanese patients with type 2 diabetes mellitus. Metabolism 56: 693-698, 2007.

32. Takata Y, Osawa H, Kurata M, Kurokawa M, Yamauchi J, Ochi M, Nishida W, Okura T, Higaki J and Makino H: Hyperresistinemia is associated with coexistence of hypertension and type 2 diabetes. Hypertension 5: 534-539, 2008.

33. de Luis DA, Sagrado MG, Conde R, Aller R, Izaola O, de la Fuente B, Castrillón JL and Romero E: Relation of resistin levels with cardiovascular risk factors, insulin resistance and inflammation in naïve diabetes obese patients. Diabetes Res Clin Pract 89: 110-114, 2010.

34. Al-Daghri N, Chetty R, McTernan PG, Al-Rubean K, Al-Attas O, Jones AF and Kumar S: Serum resistin is associated with C-reactive protein and LDL cholesterol in type 2 diabetes and coronary artery disease in a Saudi population. Cardiovase Diabetol 4: 1-6, 2005.

35. Lau CH and Muniandy S: Novel adiponectin-resistin (AR) and insulin resistance (IRAR) indexes are useful integrated diagnostic biomarkers for insulin resistance, type 2 diabetes and metabolic syndrome: a case control study. Cardiovasc Diabetol 10: 8, 2011.

36. Esteghamati A, Alamdari A, Zandieh A, Elahi S, Khalilzadeh O, Nakhjavani M and Meysamie A: Serum visfatin is associated with type 2 diabetes mellitus independent of insulin resistance and obesity. Diabetes Res Clin Pract 91: 154-158, 2011.
37. Alghasham AA and Barakat YA: Serum visfatin and its relation to insulin resistance and inflammation in type 2 diabetic patients with and without macroangiopathy. Saudi Med J 29: 185-192, 2008.

38. Chang YH, Chang DM, Lin KC, Shin SJ and Lee YJ: Visfatin in overweight/obesity, type 2 diabetes mellitus, insulin resistance, metabolic syndrome and cardiovascular diseases: a metaanalysis and systemic review. Diabetes Metab Res Rev 27: 515-527, 2011.

39. El-Mesallamy HO, Kassem DH, El-Demerdash E and Amin AI: Vaspin and visfatin/Nampt are interesting interrelated adipokines playing a role in the pathogenesis of type 2 diabetes mellitus. Metabolism 60: 63-70, 2011.

40. Sandeep S, Velmurugan K, Deepa R and Mohan V: Serum visfatin in relation to visceral fat, obesity, and type 2 diabetes mellitus in Asian Indians. Metabolism 56: 565-570, 2007.

41. Shaker O, El-Shehaby A, Zakaria A, Mostafa N, Talaat S, Katsiki $\mathrm{N}$ and Mikhailidis DP: Plasma visfatin and retinol binding protein-4 levels in patients with type 2 diabetes mellitus and their relationship to adiposity and fatty liver. Clin Biochem 44: 1457-1463, 2011.

42. Chen MP, Chung FM, Chang DM, Tsai JC, Huang HF, Shin SJ and Lee YJ: Elevated plasma level of visfatin/pre-B cell colonyenhancing factor in patients with type 2 diabetes mellitus. J Clin Endocrinol Metab 91: 295-299, 2006.

43. Pereira EC, Ferderbar S, Bertolami MC, Faludi AA, Monte O, Xavier HT, Pereira TV and Abdalla DS: Biomarkers of oxidative stress and endothelial dysfunction in glucose intolerance and diabetes mellitus. Clin Biochem 41: 1454-1460, 2008.

44. Krzyzanowska K, Mittermayer F, Wolzt M and Schernthaner G: ADMA, cardiovascular disease and diabetes. Diabetes Res Clin Pract 15: 122-126, 2008.

45. Kanazawa I, Yano S, Notsu Y, Yamaguchi T, Nabika T and Sugimoto T: Asymmetric dimethylarginine as a risk factor for cardiovascular disease in Japanese patients with type 2 diabetes mellitus. Clin Endocrinol (Oxf) 74: 467-472, 2011

46. Yasuda S, Miyazaki S, Kanda M, Goto Y, Suzuki M, Harano Y and Nonogi $\mathrm{H}$ : Intensive treatment of risk factors in patients with type-2 diabetes mellitus is associated with improvement of endothelial function coupled with a reduction in the levels of plasma asymmetric dimethylarginine and endogenous inhibitor of nitric oxide synthase. Eur Heart J 27: 1159-1165, 2006.

47. de Luis DA, Fernandez N, Arranz ML, Aller R, Izaola O and Romero E: Total homocysteine levels relation with chronic complications of diabetes, body composition, and other cardiovascular risk factors in a population of patients with diabetes mellitus type 2. J Diabetes Complications 19: 42-46, 2005.

48. Akalin A, Alatas O and Colak O: Relation of plasma homocysteine levels to atherosclerotic vascular disease and inflammation markers in type 2 diabetic patients. Eur J Endocrinol 158: 47-52, 2008.

49. Wang Z, Pini M, Yao T, Zhou Z, Sun C, Fantuzzi G and Song Z: Homocysteine suppresses lipolysis in adipocytes by activating the AMPK pathway. Am J Physiol Endocrinol Metab 301: 703-712, 2011.

50. Li Y, Jiang C, Xu G, Wang N, Zhu Y, Tang C and Wang X: Homocysteine upregulates resistin production from adipocytes in vivo and in vitro. Diabetes 57: 817-827, 2008. 